\title{
Software
}

\section{La gestión de recursos electrónicos en bibliotecas: la oferta de Innovative}

\author{
Por Ricardo Eíto-Brun
}

\begin{abstract}
Resumen: La gestión de los recursos-e se ha convertido en una nueva necesidad para bibliotecas y centros de documentación. El número de los que una biblioteca debe controlar y a los que se debe dar acceso crece continuamente. A la par se necesitan herramientas que hagan posible un control efectivo de información sobre licencias, solapamiento entre bases de datos, contactos, aspectos técnicos, etc. En este texto se describe la oferta de la empresa Innovative Interfaces para la gestión de los recursos-e. Su completa oferta incluye distintos productos integrados entre sí, y que también pueden adoptarse de forma individual e interactuar con sistemas de otros fabricantes. Entre ellos destaca el sistema ERM (Electronic Resources Management), el sistema de resolución de enlaces WebBridge, el metabuscador ResearchPro y el novedoso Encore, con el que se reformula la forma de acceder a la información tradicionalmente usada en los opacs. Como complemento a estos productos, Innovative Interfaces ha lanzado el servicio CASE, que ofrece datos de cobertura de bases de datos y revistas-e a las bibliotecas, con servicios complementarios como descarga de registros marc para su integración en el opac.
\end{abstract}

Palabras clave: Recursos-e, Colección electrónica, ERM, Innovative Interfaces, Metabuscadores, Búsqueda federada, Sistemas de resolución de enlaces, OpenURL, Sushi.

\section{Title: Electronic resource management in libraries: what Innovative offers}

Abstract: The management of electronic resources has become a crucial need for libraries and documentation centres. The number of e-resources that a library must manage and give access to is continually increasing. Simultaneously, new tools are needed for effective control of information about licenses, overlap between databases, contacts, and technical issues related to e-resources. The Innovative Interfaces e-resources management software, which includes a variety of integrated products that can also be deployed individually with a high level of interoperability with other companies products. We highlight Electronic Resources Management (ERM), the WebBridge Link Resolver, ResearchPro (a federated search engine) and the innovative Encore, which has reformulated the traditional opac approach to information access. Innovative Interfaces has also launched the CASE service, which offers coverage data about e-journals and e-resources, with additional services like marc records for e-resources to facilitate their integration in the catalogue.

Keywords: Electronic resources, Electronic collection, ERM, Innovative Interfaces, Metasearching, Federated search, Link resolvers, OpenURL, Sushi.

Eíto-Brun, Ricardo. "La gestión de recursos electrónicos en bibliotecas: la oferta de Innovative”. En: El profesional de la información, 2008, mayo-junio, v. 17, n. 3, pp. 347-358.

DOI: 10.3145/epi.2008.may.13

LA GESTIÓN DE LOS RECURSOS ELECTRÓNICOS es uno de los grandes retos que bibliotecas y centros de documentación han tenido que afrontar en los últimos años. Con el término recursos-e se hace referencia a aquellos materiales cuya principal característica es estar dispo- nibles en formato digital, tanto si se almacenan dentro de los sistemas informáticos de la institución, como si se accede a ellos remotamente a través de una red.

Incluyen monografías y revistas publicadas en formato electrónico, sitios web y bases de datos. Estos dos tipos reciben el nombre de recursos continuos actualizables, para señalar el hecho de que sus contenidos no se actualizan en forma de nuevos números -como sucede con las revistas- sino que se incrementan en su conjunto de forma progresiva o continua, sin poder diferenciar en su estructura algo similar al concepto de número 
o volumen utilizado con las publicaciones periódicas.

La incorporación de estos materiales trajo consigo la necesidad de replantearse la forma en que las bibliotecas los seleccionan, adquieren, describen y ponen a disposición de sus usuarios. Si en rasgos generales existen unos principios comunes en la gestión de los documentos tradicionales y los electrónicos, las características de éstos han obligado a reconsiderar algunos aspectos.

Temas como la posesión frente al acceso, la complejidad de las políticas de licencias y las dificultades de establecer su precio han ocupado numerosas páginas en la bibliografía académica y profesional. Los aspectos relacionados con su descripción y la forma de aplicar esquemas tradicionales como marc o alternativas como los metadatos Dublin Core, MODS (Metadata Object Description Standard) también han sido ampliamente tratadas en distintas monografías y encuentros de trabajo. Otro tema debatido es la responsabilidad sobre la pre- servación futura de los contenidos y cómo deben los proveedores garantizar el acceso futuro a los materiales contratados por el centro si el período de suscripción expira y no se renueva. En los siguientes apartados se describen algunos de estos asuntos.

\section{Selección y control de las adquisiciones de recursos-e}

En primer lugar la biblioteca necesita registrar y mantener información del proceso de selección y adquisición.

Los elevados costes y los complejos procesos de negociación de licencias obliga a mantener un estricto control sobre los títulos contratados por los centros, conocer aquellos a los que se tiene acceso a través de consorcios y acuerdos con otras instituciones y evitar la duplicidad de materiales. Este problema se acentúa si consideramos las características de las bases de datos. Los proveedores suelen organizar su oferta en torno a paquetes o grupos de publicaciones electrónicas

\section{Proveedores, agregadores, editores...}

La bibliografía sobre recursos-e suele diferenciar distintos términos para referirse a los proveedores de información que ofrecen acceso al texto completo de los mismos de forma directa o indirecta. Así un proveedor puede ser el editor de una revista-e que haya organizado un servicio para acceder a los contenidos de sus propias publicaciones. Otro término usado frecuentemente es el de agregadores, con el que se hace referencia a empresas que facilitan acceso a artículos y revistas-e publicadas por distintos editores, mediando entre éstos y los usuarios finales. En ocasiones también se diferencia entre: a) los servicios que actúan como meros mediadores, ofreciendo a los usuarios una base de datos referencial, desde la cual se dirige al sitio web del editor cuando el usuario solicita el texto completo de un artículo, y b) los servicios que disponen también del texto completo de los artículos, en cuyo caso no es necesario acceder al sitio de los editores (el término agregador suele reservarse a los proveedores que utilizan este segundo modelo). En el texto hemos usado el término proveedores en general, para referirnos a cualquier organización que publique revistas-e o bases de datos a través de la web.

que se distribuyen de forma conjunta. Así, un centro puede contratar una base de datos que ofrece acceso a una colección de revistas electrónicas -o títulos- sobre una temática determinada. Algunos de estos títulos pueden también ofertarse en otros paquetes de ese mismo o de otro proveedor, con el riesgo de pagar dos veces por el mismo material.

Además de conocer con exactitud los títulos para los que se ha contratado acceso, los responsables de su selección y adquisición deben disponer de información actualizada sobre períodos de suscripción -normalmente el acceso a los productos-e se suscribe por un tiempo limitado, que se podrá renovar posteriormente-, fechas en las que se debe informar al proveedor de la renovación o cancelación de las suscripciones y datos de contacto de los proveedores (para obtener soporte técnico, discutir aspectos contractuales, etc.). La información sobre publicacione digitales en período de prueba o evaluación también debe ser registrada y conocida por el personal de la biblioteca y por los usuarios implicados.

\section{Puesta a disposición}

La contratación del acceso debe seguirse de su puesta a disposición para los usuarios. Esto se completa en una serie de actividades:

- catalogación,

- publicación de estas descripciones en el opac tradicional o mediante página o directorios web, $\mathrm{y}$

- resolución de aspectos técnicos relativos a la configuración del acceso.

Aquí caben distintas alternativas, como acceder según nombres de usuario y contraseñas, o el sistema más habitual basado en el filtrado de direcciones ip. Este modelo hace que sólo pueden acceder los usuarios conectados a un ordenador de la red institucional. El sitio 
web del proveedor donde se alojan los contenidos comprobará desde qué ordenador se está cursando la petición; si ésta se hace desde un ordenador conectado a la red informática de la institución que lo ha contratado, el proveedor establecerá la sesión y permitirá la consulta. En caso contrario la petición será denegada. La forma en que se ha configurado y negociado el acceso a los recursos electrónicos remotos debe ser conocida en todo momento por el personal de la biblioteca encargado de su gestión. Si la biblioteca opta por mantener los contenidos contratados dentro de su red institucional (en este caso no se haría a través de una conexión remota sino mediante la red corporativa del centro en la que se mantienen copias electrónicas de los documentos), también se debe proceder a la organización de la infraestructura técnica necesaria.

La catalogación y publicación de las fuentes electrónicas ha venido realizándose de dos formas: a) directorios publicados en la web del centro y b) integrando las descripciones dentro del opac tradicional. Partidarios y detractores de una $\mathrm{u}$ otra opción han expuesto sus ventajas e inconvenientes, aunque como conclusión general suele afirmarse que es la combinación de las dos modalidades la que ofrece resultados óptimos para los usuarios.

En el caso de los directorios, los usuarios pueden consultar las llamadas listas A-Z, páginas web donde las fuentes electrónicas contratadas se presentan en orden alfabético. Para cada título en el listado, se suele indicar una breve descripción de su contenido, materias de las que trata, y su cobertura cronológica (la que tiene contratada el centro). Desde aquí el usuario puede hacer clic en el título en cuestión para navegar al sitio web del proveedor y comenzar su sesión de trabajo. Normalmente se complementa con otra lista de recursos agrupados por

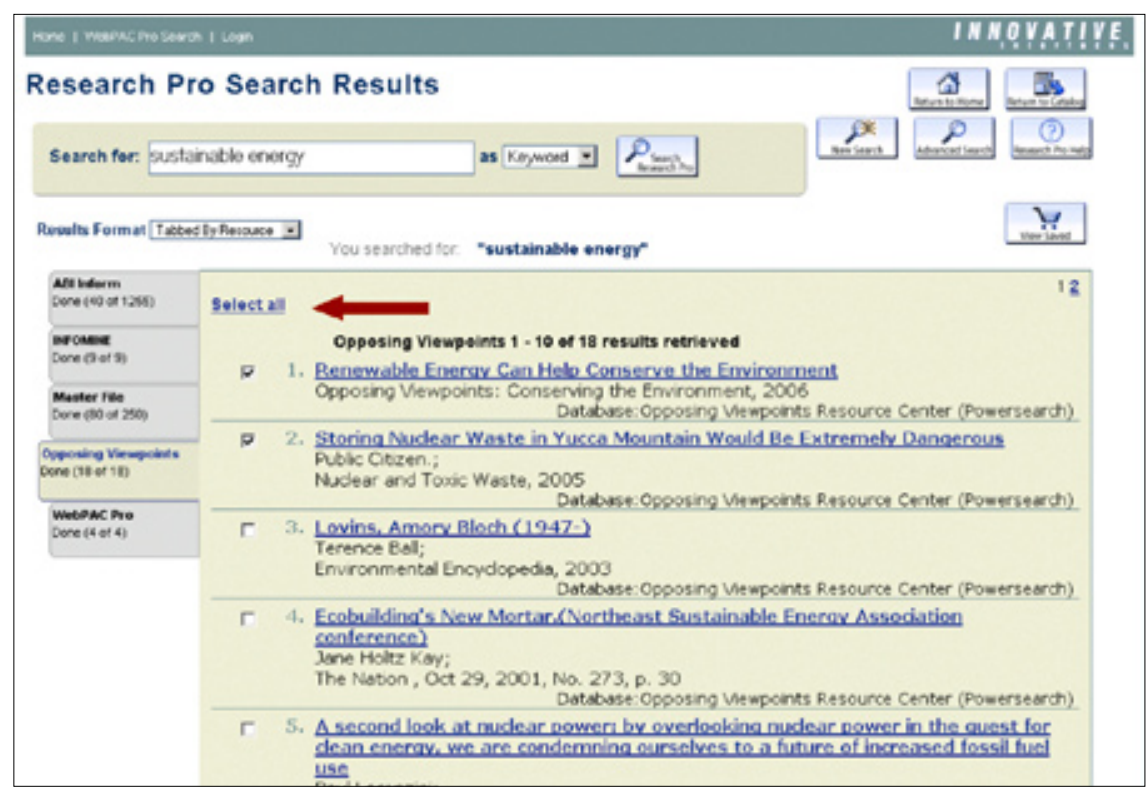

Interfaz de usuario de ResearchPro

temas y ofrece la posibilidad de buscar por palabras contenidas en los títulos o descripciones de los recursos-e.

Ofrecen un mecanismo de acceso rápido y eficiente aunque se debe considerar la posibilidad de incorporar al catálogo tradicional registros para estas fuentes, para que las búsquedas en el opac muestren al lector todos los títulos disponibles a través del centro, electrónicos o no, sean parte de las colecciones locales o de las disponibles remotamente. Esto obliga a dar de alta en el catálogo registros marc para los contenidos digitales. Como conclusión, para ofrecer al usuario las mayores garantías, la biblioteca debe realizar el doble trabajo de mantener las listas A-Z a la par que los registros catalográficos del opac.

\section{Mantenimiento de la cobertura de las publicaciones}

En el caso de las revistas electrónicas, los bibliotecarios también deben mantener información actualizada sobre el estado de la colección y la disponibilidad de nuevos números conforme éstos se publican (con las bases de datos de revistas-e se presenta una situación similar). El control ejercido con el tradicio- nal kárdex utilizado en las bibliotecas para controlar la recepción de los números de las publicaciones impresas también es necesario con las digitales. De esta forma no se registrará la llegada de los nuevos números al centro, sino la disponibilidad en línea de un nuevo número en el sitio remoto del proveedor. En centros donde se trabaja con un alto número de revistas-e y bases de datos de distintos proveedores, mantener esta información actualizada se convierte en un esfuerzo y costes sumamente elevados. Es también una actividad realmente complicada incluso si se cuenta con una estrecha colaboración por parte de los proveedores. Por otra parte puede darse el caso de que éstos modifiquen los títulos distribuidos a través de una base de datos, con el consiguiente problema para conocer qué está realmente disponible para los usuarios del centro.

En respuesta a las dificultades que acarrea el mantenimiento de la cobertura de los recursos electrónicos y controlar su evolución, en los últimos años han surgido empresas cuya principal actividad es mantener y distribuir a los centros usuarios de este material, información sobre la cobertura de las bases de datos de distintos proveedores y de 


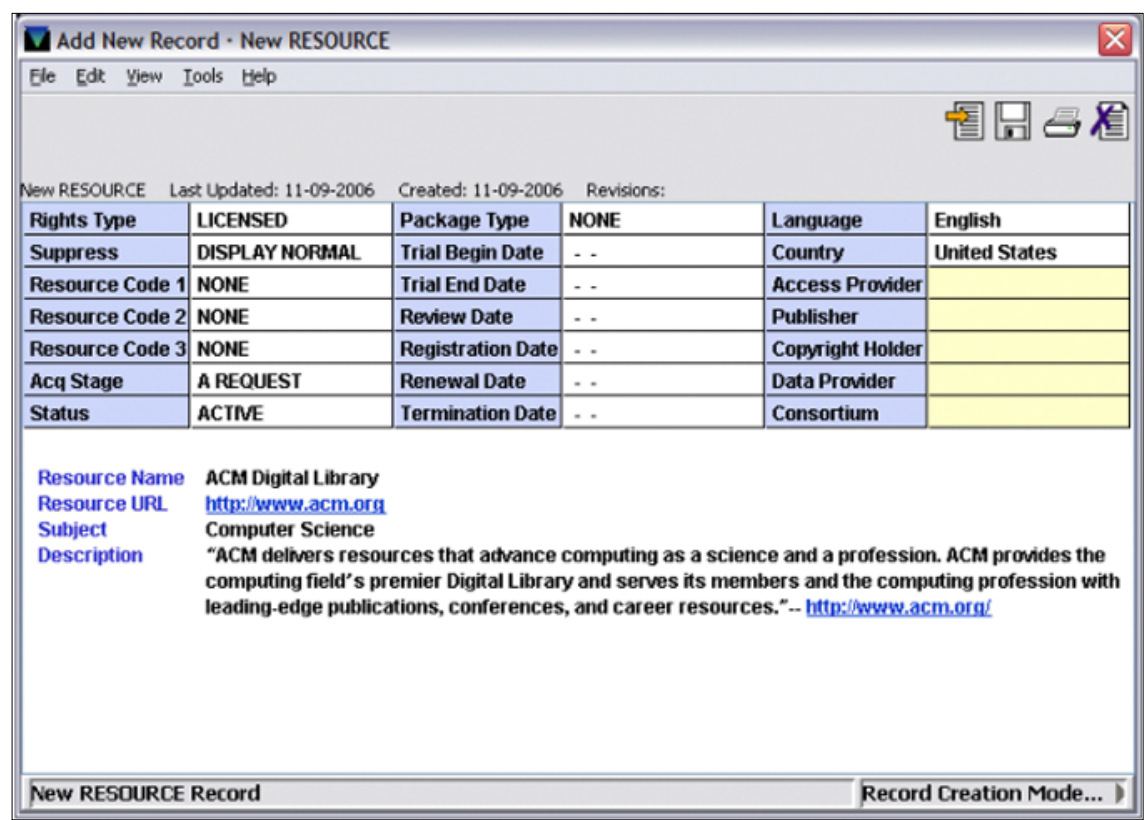

Ventana de ERM con información sobre un recurso-e

las actualizaciones que se producen en sus contenidos y títulos. Facilitan listas A-Z con los contenidos que tienen suscritos, y se encargan de su actualización, descargando a las bibliotecas de esta actividad. Normalmente, las listas A-Z son páginas personalizadas para cada centro (tanto en sus contenidos como en su aspecto y formato), hospedadas en los servidores web de esas empresas, que actúan como mediadoras entre proveedores y centros. También suelen facilitar registros en formato marc para su integración en los opacs.

Entre esas empresas destacan SerialsSolutions, Gold Rush o TDNet (SFX ofrece un servicio similar llamado Citation Linker). Obviamente los proveedores de recursose también ofertan servicios similares para las bibliotecas, como por ejemplo el Ebsco A-to-Z Service. En numerosos sitios web de centros bibliotecarios universitarios españoles podemos ver que sus listados de recursos electrónicos nos redirigen a páginas alojadas en algunas de estas empresas. Innovative ofrece, en complemento con sus herramientas, el servicio CASE (Content Access Service) tanto para datos de cobertura (Coverage Data Service) incluyendo el soporte a los servicios A-Z, como para datos marc (Marc Record Service).

\section{Uso de los recursos-e}

Una vez se han puesto a disposición de los usuarios, la biblioteca debe esforzarse por conocer el uso real que se hace de los mismos y evaluar la rentabilidad de las suscripciones. Tienen un coste elevado y su uso constituye un aspecto decisivo a la hora de decidir renovarlas o cancelarlas. Los estudios de utilización también permiten evaluar la rentabilidad de suscribir determinados paquetes de revistas-e o servicios. Los proveedores suelen ofrecer acceso conjunto a publicaciones sumamente atractivas frente a otras que pueden resultar menos interesantes para la comunidad de usuarios. Disponer de un alto número de títulos a través de un mismo paquete puede resultar práctico para la biblioteca, pero quizás el uso real que se haga de ellos no justifique una inversión que inicialmente resultaba atractiva. En resumen, se debe conocer con la mayor exactitud posible el uso que los usuarios hacen de la colección de recursos digitales.

En este área también se han de- sarrollado importantes iniciativas, como el proyecto Counter o el estándar Sushi (Standardized Usage Statistics Harvesting Initiative), dos proyectos complementarios. Por una parte, el primero se constituye como una forma de medir el uso que se hace de los recursos-e. Para ello establece una definición precisa de las actividades que se van a medir (búsquedas, accesos, descargas, etc.) y la forma en la que éstas acciones deben totalizarse. Se definen también unos informes normalizados para medir el uso que se hace en distintos períodos de tiempo.

En Counter, la responsabilidad de obtener las métricas recae en los proveedores. Para evitar posibles manipulaciones de los datos (se podría caer en la tentación de informar a las bibliotecas de un uso mayor del que realmente están haciendo los usuarios), Counter establece un proceso de auditoría realizado por una tercera parte, independiente del proveedor. En este proceso de auditoría se verificará la forma en que éste obtiene las medidas para asegurar que son correctas y se han conseguido siguiendo las pautas dictadas por esta iniciativa. En la actualidad los principales proveedores de bases de datos y recursos-e han declarado su conformidad con Counter y ofrecen a sus clientes la posibilidad de obtener este tipo de informes de uso descargándolos del sitio web del proveedor (normalmente en formato Microsoft Excel). La biblioteca cuenta así con la información necesaria para conocer qué títulos se consultan con mayor asiduidad, y qué bases de datos constituyen las principales fuentes de información para sus usuarios. Una de sus grandes ventajas es que todos los que aplican este método totalizarán los datos de la misma forma, lo que asegura que son comparables y se han obtenido siguiendo un mismo procedimiento.

Respecto a Sushi se trata de una iniciativa complementaria a 


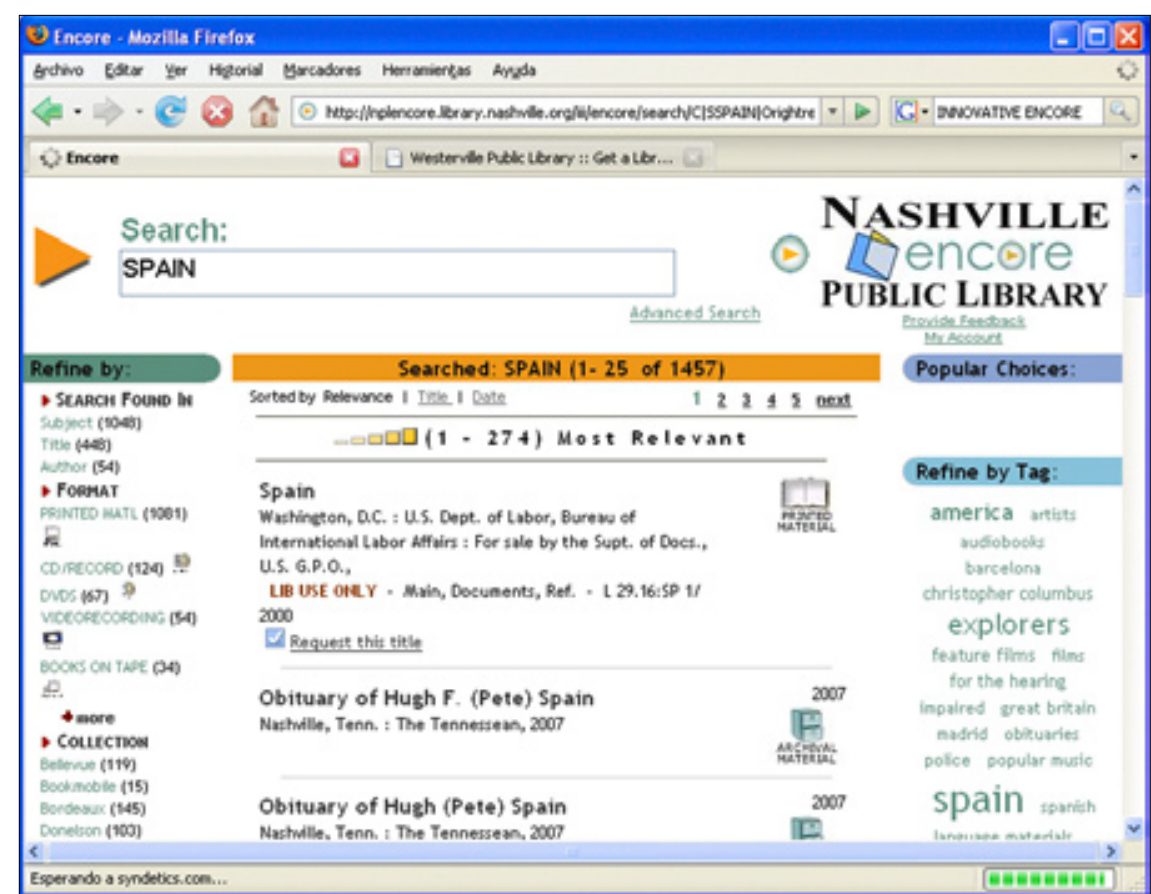

Encore constituye una aproximación novedosa para la explotación de los fondos de información

\section{La copia apropiada}

OpenURL es un mecanismo genérico para enviar metadatos a través de la web entre servicios de información. El estándar permite no sólo enviar información sobre los metadatos del recurso. También se podría enviar en una petición OpenURL datos sobre el tipo de usuario y el contexto en el que está realizando su trabajo. De esta forma el servidor de enlaces contaría con más información sobre el tipo de usuario, y podría así descartar -por poner un ejemplo- aquellos recursos a los que no tiene capacidad de acceder.

Una práctica intuitiva cuando se codifica una referencia bibliográfica en una página web sería hacer que el enlace apuntase a la página del editor donde se encuentra el texto completo. Sin embargo, puede darse el caso de que el usuario del centro no tenga acceso a ese documento, aunque sí puede tener acceso a ese mismo contenido a través de la base de datos de un agregador (ésta sería la copia apropiada). Con OpenURL, el servidor de enlaces recibe los metadatos necesarios para poder identificar cuál es la más apropiada para cada usuario.

Debemos considerar también que el destino de un enlace puede ser un servicio gratuito o de pago, un servicio donde el texto del documento esté disponible en línea o un servicio de obtención de documentos. Los servidores de enlaces deben ofrecer la posibilidad de discriminar en estos casos cuál es la opción preferible, y hacer que los usuarios seleccionen la adecuada.
Counter que establece un mecanismo para descargar los informes Counter de los sitios web de los proveedores de forma automática y desatendida. Su desarrollo se inició en junio de 2005 por un grupo de trabajo formado por la Cornell University, Ebsco, Ex Libris, Innovative, Swets y la Washington University, a los que posteriormente se añadió Thomson Scientific. Su finalidad es que sea una aplicación informática la que se encargue de conectarse regularmente a los sitios web de distintos proveedores y descargar los informes correspondientes al centro. De esta forma el bibliotecario no tendrá que dedicar tiempo a descargar uno tras otro los informes de los distintos proveedores, y puede centrar su atención a realizar el análisis de los datos. Se convirtió en la norma norteamericana $Z 39.93$ en julio de 2007.

\section{Nuevas tecnologías para la gestión}

La gestión de colecciones electrónicas ha supuesto la necesidad de desarrollar tecnologías complementarias a las que tradicionalmente venían usándose en las bibliotecas. Hoy es frecuente oír hablar de servidores de enlazado, búsquedas federadas y de protocolos o estándares como OpenURL.

\section{"La gestión de activos-e ha supuesto la necesidad de tecnologías complementarias a las que tradicionalmente venían usándose en las bibliotecas"}

Los servidores de enlazado son sin duda alguna una de las ideas más atractivas desarrolladas en los últimos años en el área de la documentación. Resuelven uno de los aspectos más complejos de la ges- 
tión de contenidos electrónicos: la multiplicidad de accesos a un mismo título y el problema de la copia apropiada.

Para introducir brevemente el propósito de estos servidores, plantearemos un escenario habitual en muchos centros. Supongamos que una biblioteca cuenta con distintas suscripciones a bases de datos de revistas electrónicas y que una de estas publicaciones está disponible a través de tres bases de datos diferentes, posiblemente con distintas coberturas y derechos de uso en cada una de ellas.

Un sistema de enlazado permitiría al usuario de un opac (o de una lista A-Z), hacer clic sobre el título y obtener una nueva página donde se le indica a través de qué proveedores y de qué bases de datos tiene acceso a él, su cobertura y derechos de uso. Con esta información el usuario puede seleccionar la opción que le resulte más conveniente y navegar al sitio web del proveedor seleccionado.

Esta interacción, que a priori puede parecernos algo trivial, encierra una importante complejidad técnica. En primer lugar es necesario conocer a través de qué bases de datos y proveedores está disponible la revista en cuestión, su cobertura y condiciones de acceso en cada caso (esta información es muy similar a los datos que facilitan los proveedores de listas A-Z antes citados: Serials Solutions, TDNet, CASE de Innovative Interfaces, etc.).

Por otra parte el sistema desde el cual el usuario realiza su consulta debe ser capaz de acceder a la información anterior y de alguna forma se le tienen que presentar los enlaces correspondientes. Finalmente el sistema del proveedor debe tener la opción de responder a la petición de acceso que recibe cada vez que el usuario hace clic en un enlace que le dirija a su sitio web. Es necesario contar con una utilidad informática que medie entre la aplicación ori-

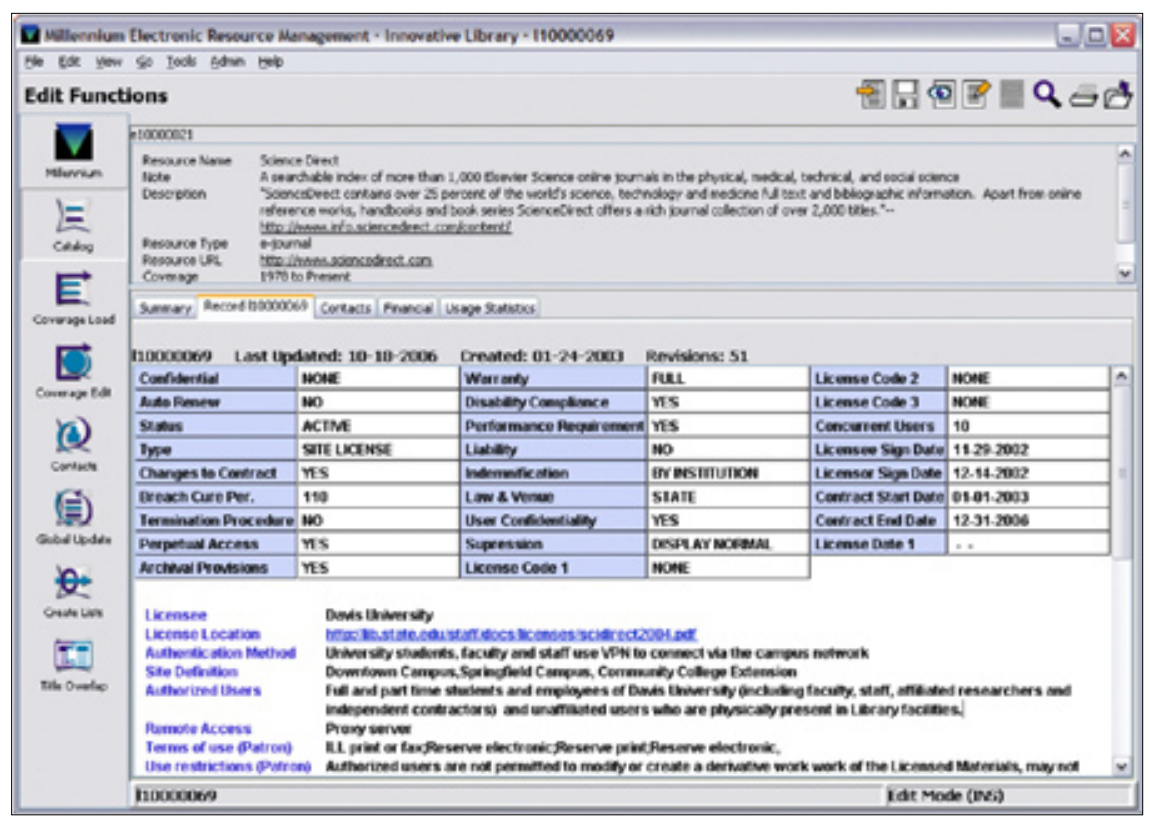

ERM permite gestionar información sobre las licencias de uso de los recursos-e

gen con la que interactúa el usuario final, y las aplicaciones y bases de datos de los proveedores que ofrecen acceso al texto completo de los artículos y revistas-e. Esa herramienta intermedia es el servidor de enlaces.

La dificultad estriba en que tanto el origen de los mismos (es decir, los opacs o listas A-Z en los que busca el usuario) como los sistemas de los proveedores son dispares, y a priori desconocidos unos de otros, por lo que establecer enlaces directos sería imposible. Además se debe contar con una forma normalizada de enlazar con los títulos ofertados por los distintos proveedores de los recursos-e. Aquí entra en juego el estándar OpenURL.

Su origen está en el trabajo de Herbert van de Sompel iniciado en 1999, y se aprobó como estándar norteamericano ANSI Z39.88 en el año 2005 (antes de esta fecha, OpenURL se había convertido en un auténtico estándar de facto). Establece una forma de hacer referencia a un recurso-e disponible en la web, usando para ello sus metadatos. Podemos decir que un vínvulo basado en este sistema es un enlace web en el que en lugar de utilizar la dirección url se usan sus metadatos para hacerle referencia. Un ejemplo para una revista electrónica podría ser éste:

http://sfxserver.uni.edu/ sfxmenu? issn $=1234-5678 \&$ date $=1$ $998 \&$ volume $=12 \&$ issue $=2$

Vemos que junto al nombre del servidor al que se dirige la petición se añaden metadatos sobre el recurso-e: su código ISSN, fecha, volumen y número. Como estos metadatos constituyen el contenido básico de un enlace OpenURL, se suele decir que este estándar constituye una forma de "transportar metadatos a través de la web".

El enlace OpenURL anterior no se dirigirá al sitio web del proveedor del recurso, sino a una aplicación informática complementaria llamada servidor de enlaces (link resolver). El servidor de enlaces interpretará los metadatos incluidos en la petición, comprobará la disponibilidad del recurso solicitado en los distintos proveedores de recursos-e, y devolverá al usuario una página web con el listado de accesos (esta vez, los enlaces apuntarán a los sitios web y bases de datos de cada uno de los proveedores que se hayan identificado).

El servidor de enlaces establece así un puente entre una aplica- 


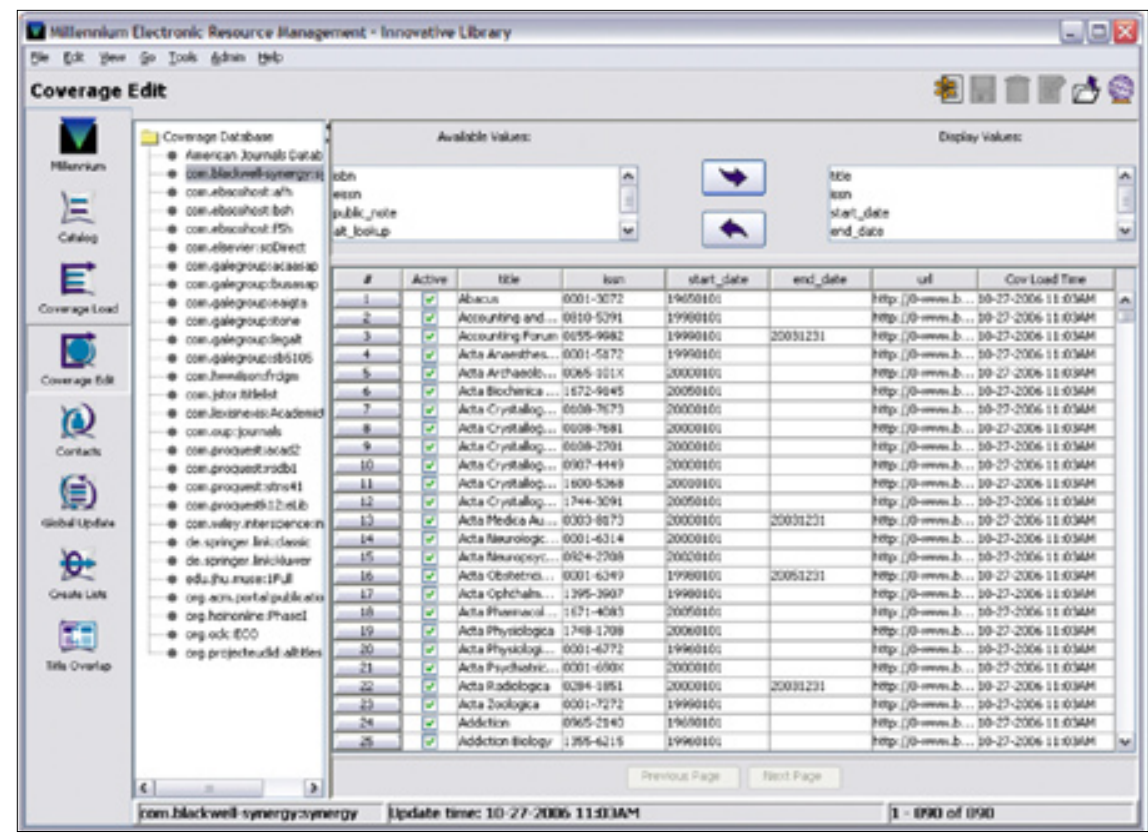

Desde ERM se puede gestionar la lista de revistas-e accesibles a través de distintas bases de datos

\section{"Como los metadatos del recurso-e constituyen el contenido básico de un enlace OpenURL, se suele decir que este estándar constituye una forma de 'transportar metadatos a través de la web"'}

ción origen (normalmente un opac o una lista $\mathrm{A}-\mathrm{Z}$, aunque podría ser cualquier otra, incluso una base de datos de un tercero) y las bases de datos de los distintos proveedores que ofrecen acceso a los recursos-e o a algún servicio relacionado con ellos (texto completo, obtención de documentos, préstamo interbibliotecario, etc.). Su funcionamiento se basa en:

- la capacidad de recibir e interpretar peticiones OpenURL procedentes de la aplicación origen,

- la disponibilidad de información sobre la cobertura de los títulos en los distintos servicios y bases de datos contratados por el centro, $\mathrm{y}$

- la capacidad de presentar al usuario enlaces que apunten a los documentos relevantes de distintas bases de datos y proveedores. Los sistemas de búsqueda federada tienen como interfaz una página de consulta que permite consultar simultáneamente bases de datos y revistas-e hospedadas en sitios web de distintos proveedores, recursos internos, etc. Los usuarios pueden interactuar y consultar múltiples bases de datos desde un único punto.

El objetivo es redirigir la petición del usuario a múltiples sitios, recibir los resultados e integrarlos en una única lista en la que idealmente se eliminarán posibles duplicados. Para que esto sea posible, los proveedores deben exponer al exterior sus catálogos y dar la posibilidad de recibir búsquedas a su sistema desde otras aplicaciones informáticas.

$\mathrm{Su}$ funcionamiento puede verse como algo similar a un cliente Z39.50 usado para consultar simultáneamente varios catálogos, aunque existe una diferencia importante: los proveedores de recursos-e y bases de datos pueden exponer sus datos usando distintos protocolos o normas, no sólo Z39.50. En este sentido contamos con iniciativas que han alcanzado la madurez, como SRW/SRU (Search and Retrieval Web/URL) de la Library of Congress norteamericana y MetaSearch de NISO (US National Information Standards Organization) basada en el anterior. $S R W / S R U$ puede definirse como una adaptación de Z39.50 a la web, basada en el intercambio de peticiones y respuestas $\mathrm{xml}$, que simplifica en gran medida la programación de este tipo de soluciones. Aparte de soportar protocolos normalizados como Z39.50 o $S R W / S R U$, los meta-buscadores pueden implementar otros de tipo propietario, específicos de cada proveedor, normalmente basados en el intercambio de peticiones $\mathrm{y}$ respuestas en $\mathrm{xml}$. 


\section{Oferta de Innovative para la gestión}

Innovative ofrece una serie de aplicaciones para la gestión de recursos electrónicos y de las situaciones descritas en los apartados anteriores. A finales de 2007 la empresa publicó una actualización de sus programas para la gestión de estas fuentes, y uno nuevo, Encore, que podríamos situar próximo a los conceptos que caracterizan la llamada web 2.0.

La relación continua con grupos de usuarios y directores de bibliotecas a partir de distintos foros y encuentros de trabajo es la base de una reflexión profunda de Innovative para conocer de primera mano las inquietudes y las necesidades de los usuarios y profesionales de las bibliotecas. Por otra parte, la independencia económica de la empresa respecto a grupos de inversión elimina la presión de los resultados a corto plazo y permite sacar los productos cuando han alcanzado la madurez necesaria y han sido testados con exhaustividad.

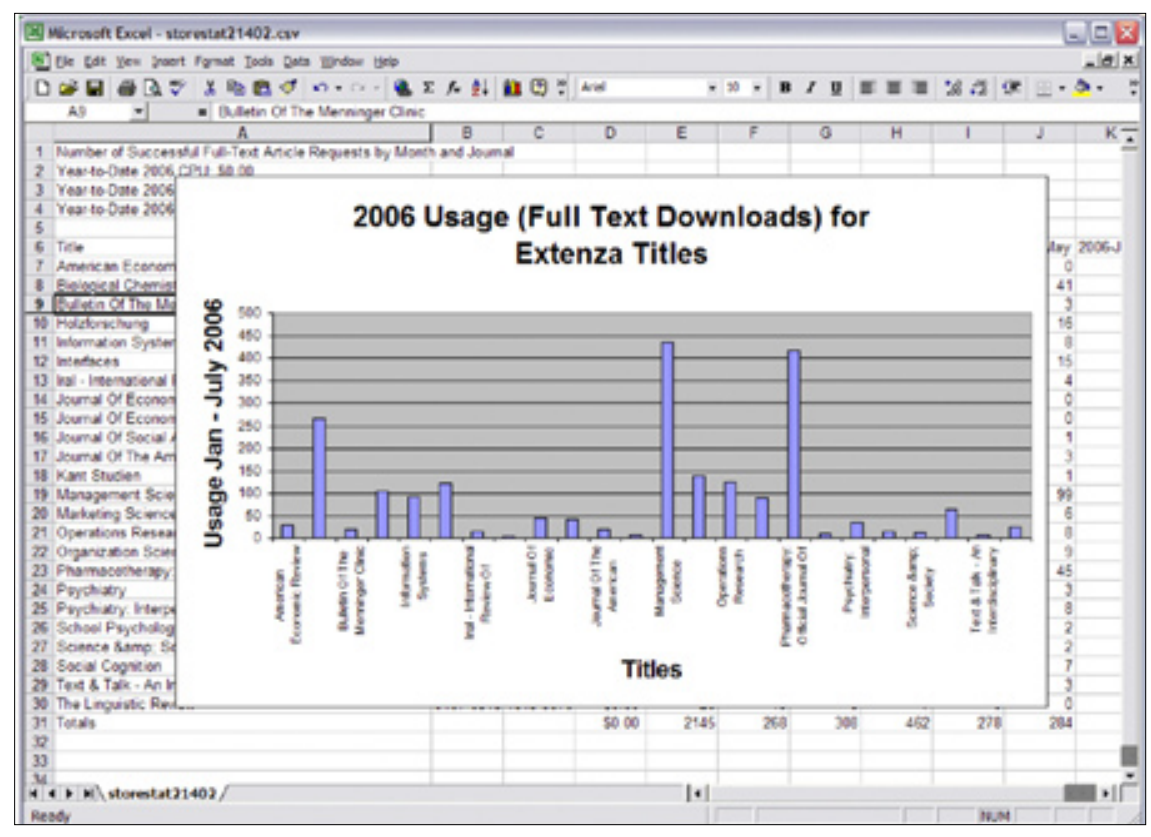

Los informes de uso de ERM son clave para la organización eficiente de la colección electrónica
La oferta de Innovative para la gestión de recursos-e incluye distintas aplicaciones:

- Servidor de enlaces WebBridge LR (Link Resolver), complementado con PathFinder.

- Metabuscador Research Pro.

- Sistema gestor de recursos

\section{$S R W / S R U$}

Son unos protocolos desarrollados por la Library of Congress norteamericana dentro de su iniciativa ZING (Z39.50 Next Generation). Establecen una serie de mensajes de intercambio -formulados en xml-entre un ordenador cliente y un servidor. Su objetivo es normalizar la forma de consultar un servidor de información remoto disponible en la web. En este sentido, SRW/SRU viene a ser una simplificación de la norma Z39.50, adaptada a las características técnicas de la web.

La diferencia entre SRW y SRU es que, en el primero, tanto las peticiones como las respuestas se envían en forma de documentos $\mathrm{xml}$, mientras que en el segundo se mandan mediante parámetros que se añaden a la url del servidor remoto. Se describe en:

http://www.loc.gov/standards/sru/

Este estándar constituye la base de la llamada NISO MetaSearch Initiative, de la que se puede encontrar más información en:

http://www.niso.org/committees/MS_initiative.html electrónicos ERM y el servicio CASE.

- Y su último lanzamiento, Encore.

Junto con éstas, debemos hacer referencia a Symposia -para la gestión de repositorios institucionalesy MetadataBuilder-para la integración en el catálogo de descripciones de fuentes codificadas en sistemas de metadatos Dublin Core y EAD $X M L$-. Otro módulo importante es Web Access Management, que actúa como un proxy para que los usuarios conectados desde fuera de la red institucional puedan consultar las bases de datos cuyo acceso está restringido a los ordenadores situados dentro de la misma. Todos estos programas pueden adquirirse separadamente, e incluso integrarse con aplicaciones de otros fabricantes.

\section{WebBridge LR y PathFinder}

Sus funciones son complementarias. El primero proporciona la tecnología de enlace que permite llevar a los usuarios a los recursos digitales apropiados, desde fuentes de información externas al catálogo. Se trata de un resolvedor de enlaces OpenURL. En muchos casos 
se requiere un enlace desde la cita al texto completo del artículo. Es posible agrupar los recursos relacionados en categorías definidas por la biblioteca como: Disponibilidad del texto completo, Fuentes de referencia, Enciclopedias o Fondos en otras bibliotecas. WebBridge puede crear estas listas de materiales relacionados desde cualquier recurso electrónico que pueda mandar una OpenURL al servidor de resolución de enlaces. Se usa una base de cobertura para comprobar la disponibilidad del contenido de texto completo. Para ello Innovative propone el componente Coverage Data Service de CASE. WebBridge LR es compatible con iniciativas como Google Scholar, DOI (Digital Object Identifier) y está integrado con ERM.

PathFinder Pro brinda enlaces desde el catálogo público a partir de búsquedas del usuario y hacia contenido electrónico relevante. Es un instrumento de descripción ( finding aid). Usa reglas sofisticadas -denominadas enlaces inteligentes (smart linking)- definidas por la biblioteca para cumplir con este objetivo en el opac y en Encore. Smart Linking capacita a la biblioteca para definir reglas específicas para mostrar los enlaces así como

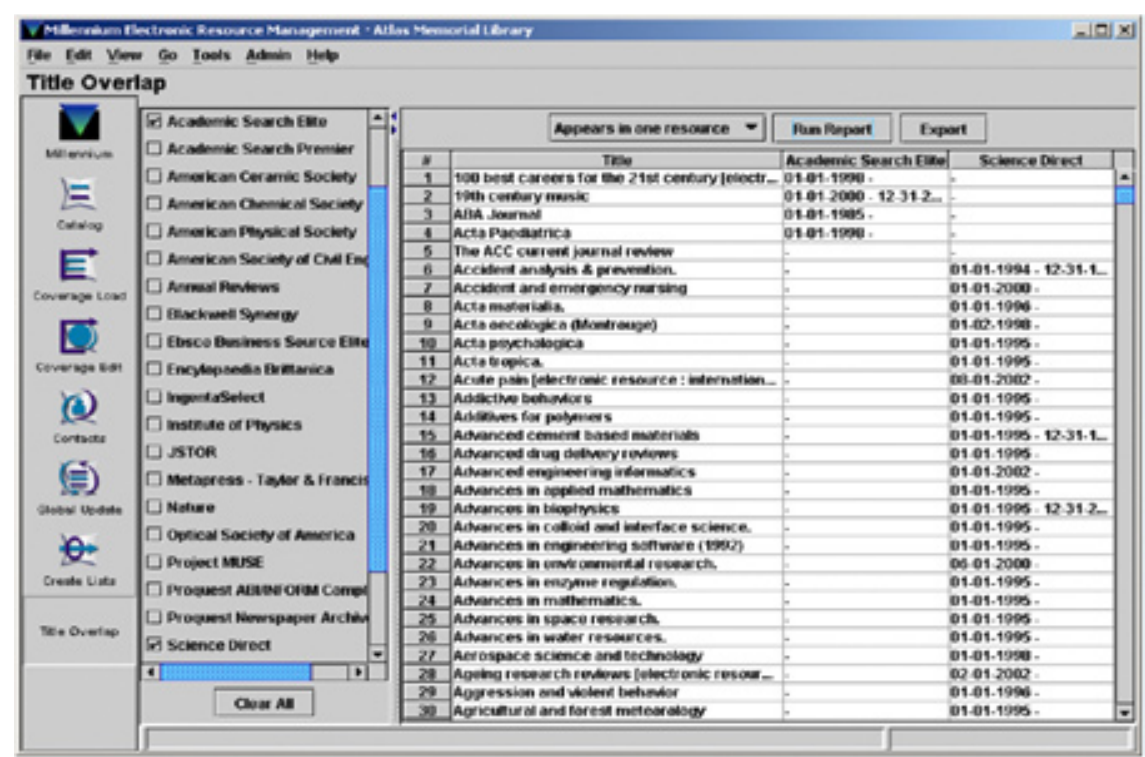

ERM permite conocer el nivel de solapamiento existente entre las distintas bases de datos, y optimizar así la inversión determinar qué campos se incluyen en las búsquedas preformateadas. De este modo, consigue incluir vínculos apropiados para elementos del registro como materia, rango de clasificación, nombre del autor, etc. Igualmente es posible enviar el campo apropiado (como la materia, el autor, el título, etc.) al recurso adecuado. PathFinder Pro habilita la generación automática de enlaces a recursos relevantes como contenido enriquecido, bases de datos de suscripciones, otras bibliotecas, base de datos en web al transferir una búsqueda existente a cualquier

\section{Recursos origen y destino en WebBridge LR (Link Resolver)}

WebBridge está configurado por defecto para interactuar con distintos fondos de información y bases de datos. Los sistemas que pueden actuar como origen de las peticiones son todos aquellos capaces de generar peticiones OpenURL. Entre ellos se encuentran: BioMed Central, Cambridge Scientific Abstracts, EbscoHost, Ei Village, The Gale Group Infotrac, OCLC First Search, ProQuest, OVID, SilverPlatter, RLG, SwetsNetNavigator, Web of Science, etc.

Los sistemas destino de los enlaces gestionados por WebBridge LR (Link Resolver) incluyen, entre otros y además de los ya citados como origen: Altavista, Baker \& Taylor Bookjackets, Ebsco EJS, Enciclopedia Británica, Google, Ingenta, Infomine, etc. recurso con una url predecible sin necesidad de reformulación de la búsqueda, en modo de flujo continuo. Está integrado con el opac (WebPac Pro), con Encore y con los módulos de plantilla desde los cuales también se puede sacar provecho de los beneficios del Smart Linking.

\section{Research Pro}

Es la evolución de MetaFind. Consiste en un metabuscador para consultar simultáneamente distintos sistemas: el propio catálogo, otros catálogos, servicios de información y bases de datos de recursos-e remotos, etc. El usuario debe teclear los términos de búsqueda, los servicios en los que quiere realizar la consulta y lanzarla. De esta forma reenvía la búsqueda a los sistemas seleccionados $\mathrm{y}$, conforme recibe los resultados, los muestra organizados en distintas secciones según su procedencia (también se puede ver una lista integrada de resultados).

La principal mejora en esta aplicación respecto a MetaFind consiste en el uso de la novedosa tecnología AJAX (Asynchronous Javascript and $X M L)$, que se traduce en una mayor rapidez en la visualización de resultados (el sistema los mues- 


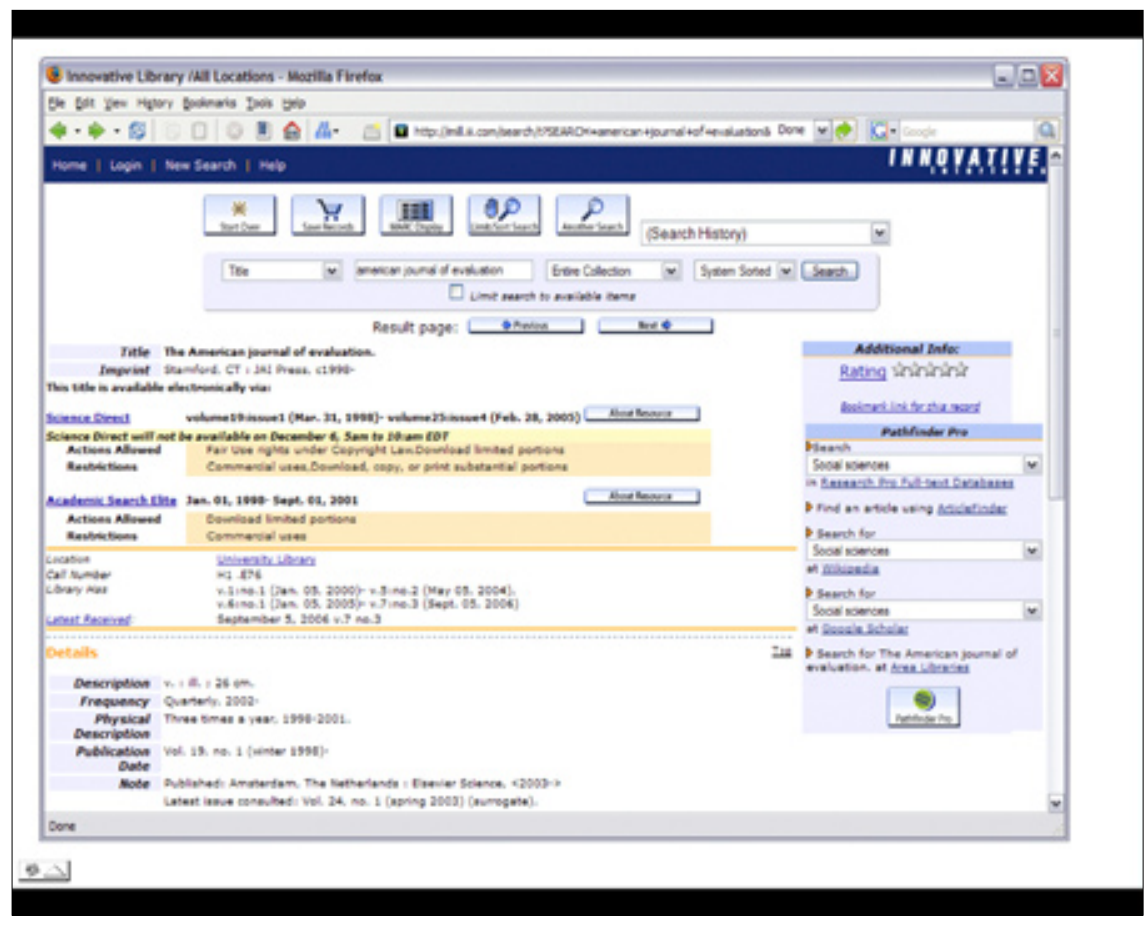

Los recursos-e catalogados desde ERM están accesibles vía web para los usuarios

tra mientras sigue completando las búsquedas). De hecho, conforme el usuario visualiza registros y documentos, Research Pro continúa recuperando datos de los servidores remotos, sin que el proceso de recuperación se interrumpa cuando el usuario decida ver un registro o lanzar una búsqueda adicional (como sucede en los sistemas de búsqueda que usamos normalmente).

También se ha facilitado en gran medida la definición de conectores entre el metabuscador y los sistemas remotos a los que se dirigen las consultas (debemos señalar que la mayor parte de conectores ya vienen predefinidos en la configuración inicial del sistema).

Además de estas mejoras técnicas, Research Pro incorpora funciones para facilitar la recuperación. El bibliotecario también puede decidir de qué forma quiere organizar y presentar las fuentes disponibles para consulta en el formulario de búsqueda. Los usuarios tienen la posibilidad de almacenar un perfil con las fuentes que quiere habilitar en todas sus consultas (para no tener que elegirlos una y otra vez en distintas sesiones de trabajo).

\section{ERM (Electronic Resource Management)}

En 2007 se publicó la versión 4 de ERM. Este programa cuenta ya con más de trescientas instalaciones en grandes bibliotecas. Es un sistema de gestión de recursos electrónicos desarrollado conforme los requisitos establecidos por ERMI (Electronic Resource Management Initiative) de la DLF (Digital Library Federation) norteamericana. Innovative fue una de las organizaciones participantes en esta iniciativa junto con otros grupos de usuarios y proveedores de sistemas informáticos.

Permite gestionar una base de datos de proveedores, recursos-e y títulos, con toda la información necesaria sobre su cobertura, derechos y condiciones de uso. La información se organiza en torno a un registro para cada proveedor $y$ base. Formará parte de este registro toda la información necesaria para la gestión del recurso-e a lo largo de su ciclo de vida: adquisición, términos de la licencia, suscripciones, personas de contacto, peticiones cursadas al soporte técnico, títulos que incluyen y su cobertura, infor- mación sobre embargos, duración de períodos de prueba, url para la descarga de informes y estadísticas de uso, etc.

Una función interesante es un informe para comprobar el solapamiento de títulos y cobertura entre bases. De hecho, el conjunto de metadatos disponible en ERM amplía los propuestos por la iniciativa ERMI.

A estas funciones se unen otras características como el acceso a la base de datos ERM mediante listas A-Z y de materias, y su plena integración con el sistema Millennium, permitiendo al usuario consultar el catálogo marc y la base de datos ERM simultáneamente, desde la interfaz web del opac.

Tanto en su uso como módulo independiente, como si se utiliza integrado con Millennium, mantiene su capacidad de interactuar con el servidor de enlaces WebBridge $L R$ mediante el que los usuarios podrán ser redirigidos a los distintos servicios desde los que tengan acceso a los títulos. Los datos sobre recursos-e gestionados en ERM tienen también visibilidad a través del metabuscador Research Pro. Además de este extenso repositorio de datos, la versión 4 de ERM ha incorporado mejoras adicionales como el soporte a la versión $1.0 \mathrm{del}$ protocolo Sushi (para la descarga automática de informes de uso de los sitios web de los proveedores), informes complementarios sobre uso y análisis de coste por materias (que se unen a los disponibles en versiones anteriores para el análisis de coste de recursos y títulos) y opciones adicionales para obtener información de cobertura desde servicios externos.

\section{CASE}

Sobre este punto, ya en versiones anteriores de ERM -concretamente desde 2006- Innovative lanzó el servicio CASE, mediante el cual ofrece datos de cobertura a las 


\section{DLF ERMI}

Este proyecto comenzó a mediados de 2002, y sus resultados se publicaron a través de la Cornell University's Web Hub for Developing Administrative Metadata for Electronic Resource Management.

\section{http://www.library.cornell.edu/cts/elicensestudy/}

En uno de los informes se propuso un ciclo de vida para los recursos-e, desde su selección hasta su puesta a disposición para los usuarios, y una serie de datos que las bibliotecas debían mantener para su gestión. Un total de trescientos metadatos y cincuenta requisitos agrupados en distintas áreas establecieron las funciones que deben implementar los sistemas informáticos para la gestión de los fondos-e. Las áreas en las que se agrupan los requisitos son: a) generales; b) relativos a la recuperación de recursos y su presentación en la web y a través del opac; c) gestión bibliográfica, donde destaca la necesidad de contar con un único punto de entrada para los datos; d) gestión de acceso desde un punto de vista técnico, y e) requisitos que el sistema debe ofrecer para el personal de la biblioteca encargado de su mantenimiento y gestión. El estudio de las características funcionales que debían incorporar los sistemas ERM también se basó en algunos programas desarrollados en universidades norteamericanas para uso interno, como el sistema Erlic (Electronic Resources Licensing and Information Center) de la Pennsylvania State University, VERA (Virtual Electronic Resource Access) del MIT, Hermes de la Johns Hopkins University o Gold Rush de Colorado Alliance of Research Libraries.

bibliotecas. Sería algo equivalente a lo que ofrecen los antes citados $\mathrm{Se}$ rialsSolutions o TDNet.

Recoge información de la cobertura de más de doscientos proveedores y bases de datos que pueden integrarse y usarse tanto desde ERM como desde WebBridge. De hecho, el sistema ERM puede adquirirse con informaciones ya cargadas desde CASE (se darán de alta en la base de datos los registros de proveedores, bases de datos y títulos correspondientes a las colecciones y servicios contratados por cada biblioteca). WebBridge puede utilizar la información sobre cobertura disponible en $C A S E$ para la resolución de enlaces.

La personalización de la base de datos para cada biblioteca se hace a través de una interfaz web -Profile Manager- donde el bibliotecario selecciona las que tiene suscritas y sus características (recordamos que los datos de cobertura de cada título en las distintas bases los facilita $C A S E$, por lo que éstos no deben darse de alta, lo que simplifica en gran medida la puesta en marcha del servicio).
También ofrece una lista A$\mathrm{Z}$ hospedada por Innovative, que puede adaptarse a la imagen institucional de cada centro. La obtención de registros marc de los títulos suscritos para su integración en el catálogo también está disponible mediante este servicio.

\section{Interfaz de consulta de Encore}

Se basa en una página de búsqueda inicial donde el usuario puede teclear sus términos de consulta. El sistema devolverá una lista de resultados ordenados por relevancia y paginados. A la izquierda se ven las facetas que permiten restringir o acotar los registros de la lista de resultados aquéllos que cumplan una serie de características: ser de un tipo determinado, pertenecer a una u otra colección, haber sido publicados en un año, escritos en un idioma particular, o aquellos donde los términos de búsqueda figuran en los campos autor, título, etc.

En la parte derecha el sistema muestra la llamada tag cloud (nube de etiquetas) consistente en un conjunto de materias o categorías asignadas al conjunto de documentos recuperados (se muestran en un tamaño u otro dependiendo del número de documentos con los que están relacionados). El usuario puede hacer clic en estas categorías para acotar los resultados de la búsqueda a aquellos registros a los que se hayan asignado las mismas. 
tificar así posibles mejoras en los interfaces de recuperación. Encore puede definirse como una interfaz de consulta (sobre un único opac o federada). Entre sus principios se encuentra el hecho de que un usuario no tiene por qué ser un experto en recuperación de información, y la necesidad de ofrecerle una experiencia basada en la inmediatez de la búsqueda (algo similar a lo que ofrecen sistemas como Google o Amazon).

Establece las bases para una mayor participación de los usuarios en torno al interfaz de consulta, al permitirles registrar comentarios y valoraciones sobre las revistas y bases de datos. Se incorpora también un sistema de tagging para que puedan etiquetar o asignar categorías o descriptores a los contenidos y mejorar así las opciones de recuperación (la asignación de descriptores o categorías por parte de los usuarios puede ser revisada por el personal de la biblioteca).

Los resultados de una búsqueda también se pueden acotar o redefinir aplicando distintas facetas o criterios (como son el formato del documento, la colección, el idioma o los descriptores que se les ha asignado). Para ofrecer mejores oportunidades de búsqueda se incorpora una función similar al “Querías decir...?" habitual en los buscadores web, que no sólo hace comprobaciones ortográficas sobre los términos propuestos por el usuario, sino que también le ofrece palabras alternativos o complementarios que pueden resultar de su interés para formular la consulta.

El mecanismo usado para ordenar los resultados aplica algoritmos propios de Innovative donde se consideran -además de los factores usados tradicionalmente para calcular la relevancia- otros como datos de circulación de los recursos, fechas de publicación, etc.

Dirigido a los usuarios finales del catálogo, Encore también incor-

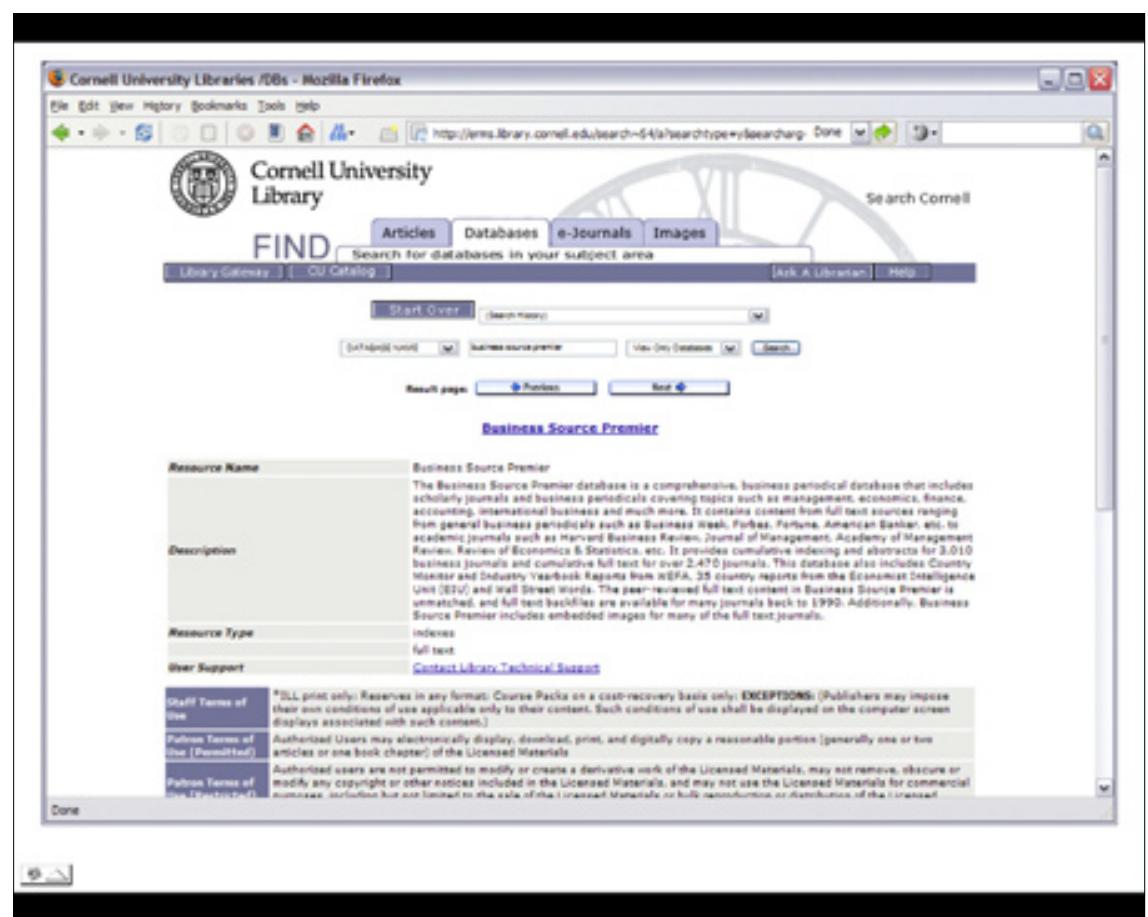

La interfaz de usuario de ERM puede adaptarse a la imagen institucional de cada biblioteca

pora un módulo donde la biblioteca puede registrar eventos a los que es posible el registro en línea, recibir notificaciones, etc.

\section{"Los sistemas integrados de gestión bibliotecaria deben explorar métodos para aumentar la participación de las comunidades de usuarios"}

En la actualidad cuenta con más de ochenta instalaciones, $y$ ha sido probado por una extensa red de socios seleccionados internacionalmente para identificar sugerencias y mejoras en la versión inicial. Innovative declara que este modelo de diseño es habitual en sus productos para asegurar que los productos responden a las expectativas de los usuarios. Con ello se quiere evitar el diseño de aplicaciones basadas en un concepto que no esté contrastado extensamente con sus necesidades reales.

\section{Conclusiones}

La oferta de productos de Innovative para la gestión de recursos-e intenta dar respuesta a muchas necesidades formuladas por bibliotecas y centros de documentación y se ha desarrollado cumpliendo los últimos estándares publicados en este área.

Con la publicación de Encore, resultado de un estudio para identificar la forma en que los usuarios interactúan con los nuevos servicios de información web, Innovative lleva a los opac estos nuevos modelos. Se trata de un paso necesario en la evolución de los sistemas integrados de gestión bibliotecaria, que además de incorporar nuevas funciones y capacidades para gestionar distintos tipos de recursos y metadatos, también deben explorar métodos para aumentar la participación de las comunidades de usuarios y resolver los problemas derivados de la sobrecarga de información que vivimos en la actualidad.

Ricardo Eíto-Brun, Universidad Carlos III de Madrid. reito@bib.uc3m.es 